\title{
SCALES OF DIVERSIFICATION AND THE ORDOVICIAN RADIATION
}

MILLER, Arnold I., Department of Geol., Univ. Cincinnati, Cincinnati, OH 45221-0013, U. S. A.

The Ordovician Radiation was arguably the most extensive episode of marine diversification, at the family level and below, in the history of life. Nevertheless, explanations for this and other major Phanerozoic diversity increases remain enigmatic. In part, this is a consequence of our general lack of knowledge concerning the ways in which these phenomena are actually played-out in more local, geographic and environmental contexts. For example, in the case of the Ordovician Radiation, it would be useful to know whether the diversity increases diagnosed in synoptic, global-scale compilations occurred randomly around the world or, alternatively, were concentrated in particular geographic regions or environmental regimes. Moreover, various avenues of recent research have suggested that temporal changes in biodiversity, including radiations, are manifestations of the same kind of general mechanism operating at different hierarchical/geographic scales, ranging from global to local.

To evaluate possible geographic and environmental selectivity associated with the Ordovician Radiation, and to determine the degree to which diversification signals were scale-transcendent, I have been compiling and analyzing a database that depicts documented occurrences of Ordovician genera around the world. Although this study is ongoing, several important aspects of the Ordovician Radiation are already emerging:

1) In evaluating temporal diversity trends, either globally or at the scale of individual paleocontinents, it is essential to first "filter-out" the effects of sample size on apparent diversification trajectories; graphs that depict raw trajectories cannot be accepted at face value.

2) After correcting for sampling effects, it is apparent that various paleocontinents exhibited overall diversity trajectories that differed from one another. In part, this reflects geological/paleoenvironmental differences from continent to continent in the Ordovician world. For example, the diversity histories of some paleocontinents were affected by their close proximity to areas of orogeny, while others were further removed from orogenic activity and were not similarly affected.

3) Diversity trends at different spatial/geographic scales did not parallel one-another. At the alpha (within-community) level, several paleocontinents exhibited diversification trajectories that were different than larger-scale, paleocontinental trends; in some cases, peak alpha diversities did not coincide with peak diversities at the paleocontinental level. In addition, there is some indication that, in contrast to patterns exhibited at other levels, beta (between-community) diversity actually decreased through the Ordovician.

Thus, in the case of the Ordovician Radiation, there are indications that diversification at different scales proceeded differently, with each scale responding to geological and biological processes perhaps unique to that scale. Thus, the "causes" of the Ordovician Radiation may be complex, though ultimately tractable. In any case, there is no indication that a single, scale-transcendent process produced the Radiation. 